\title{
Suzuki-Type Generalization of Chatterjea Contraction Mappings on Complete Partial Metric Spaces
}

\author{
Mohammad Imdad ${ }^{1}$ and Ali Erduran ${ }^{2}$ \\ ${ }^{1}$ Department of Mathematics, Aligarh Muslim University, Aligarh 202 002, India \\ ${ }^{2}$ Kirikkale University, Faculty of Arts and Sciences, Department of Mathematics, Kirikkale, 71450 Yahsihan, Turkey
}

Correspondence should be addressed to Mohammad Imdad; mhimdad@yahoo.co.in

Received 18 February 2013; Revised 21 May 2013; Accepted 22 May 2013

Academic Editor: Lingju Kong

Copyright (C) 2013 M. Imdad and A. Erduran. This is an open access article distributed under the Creative Commons Attribution License, which permits unrestricted use, distribution, and reproduction in any medium, provided the original work is properly cited.

Motivated by Suzuki (2008), we prove a Suzuki-type fixed point theorem employing Chatterjea contraction on partial metric spaces.

\section{Introduction and Preliminaries}

Historically, the idea of a complete metric space has interesting and important applications in classical analysis especially in existence and uniqueness theories on one hand while on the other hand Banach fixed point theorem [1] is one of the most useful results in nonlinear analysis. In the recent past, many authors considered the equivalence of existence results on fixed points (of mappings) by proving suitable equivalence theorems ascertaining the completeness of the underlying metric space. Kirk [2] proved that a metric space $(X, d)$ is complete if and only if every Caristi type mapping has a unique fixed point. Similarly, Subrahmanyam [3] showed that a metric space $(X, d)$ is complete if and only if every Kannan mapping has a unique fixed point. However, Banach contraction condition does not characterize the metric completeness of the underlying space. Indeed, Connell [4] gave an example of an incomplete metric space on which every contraction map has a fixed point. Despite this fact, Suzuki obtained Banach contraction principle that characterizes the metric completeness of the space using a different type of contraction. Thereafter, many authors proved different generalizations by proving different types of fixed point theorems in complete metric space, for example, Suzuki [5] proved Kannan version of a Suzuki-type generalized result wherein authors discussed contraction mappings and Kannan mappings from a different point of view while Popescu [6] attempted Chatterjea version on complete metric space.
In this continuation, Altun and Erduran proved a Suzukitype fixed point theorem using an implicit function on complete metric space [7] wherein authors obtained unified and generalized results of Suzuki-type. Finally, Kikkawa and Suzuki proved multivalued version of Suzuki-type results which generalize classical results of Markin [8] and Nadler [9]. For further details on this theme, one can be referred to [7, 10-15].

Evidently, contractions are always continuous, but Kannan mappings are not necessarily continuous. Also, one may notice that a contraction mapping is not necessarily Kannan mapping, and a Kannan mapping is not essentially a contraction mapping so that both conditions are not essentially comparable but Kannan contractions are relatively stronger than Banach contraction in certain sense. However, Chatterjea contraction is obtained by interchanging the roles of variables $x, y$ in Kannan contraction. Therefore, Chatterjea contraction is similar to Kannan contraction enabling one to infer that the mappings satisfying a Kannan contraction also satisfy the corresponding Chatterjea contraction.

In 1992, Matthews [16] introduced partial metric spaces wherein the distance of a point from itself may not always be zero. After introducing the idea of partial metric spaces, Matthews proved the partial metric version of Banach fixed point theorem. Thereafter, many authors further studied partial metric spaces and their topological properties (e.g., [17-22]) which are followed by Valero [23], Oltra and Valero [24], and Altun et al. [25] wherein authors obtained some 
generalizations of the core result of Matthews [16]. In a noted paper, Romaguera [26] extended Kirk's characterization of metric completeness [2] to partial metric spaces.

Recently, many authors proved several fixed point results in partial metric spaces. Out of such results, Suzuki-type (cf. [5]) generalizations are noted ones. Very recently, Paesano and Vetro [27] proved an analogous fixed point result for a self-mapping defined on a partial metric space (also on a partially ordered metric space) which generalizes certain noted results of Ran and Reurings [26] besides offering a characterization of partial metric 0 -completeness in terms of fixed points. This result can be viewed as an extension of Suzuki-type (cf. [5]) characterization of metric completeness.

Inspired by Paesano and Vetro [27], we prove Suzukitype fixed point result for Chatterjea contraction mappings in 0 -complete partial metric spaces wherein we show that constant $\psi(r)$ is the best for every $r$ which substantiates the genuineness and utility of our result.

In what follows, we give some relevant definitions and results from the existing literature which are relevant to our subsequent discussion.

Definition 1 (see [16]). A partial metric on a nonempty set $X$ is a function $p: X \times X \rightarrow R^{+}$such that for all $x, y, z \in X:$ (i) $x=y \Leftrightarrow p(x, x)=p(x, y)=p(y, y)$, (ii) $p(x, x) \leq p(x, y)$, (iii) $p(x, y)=p(y, x)$, (iv) $p(x, y) \leq p(x, z)+p(z, y)-$ $p(z, z)$. A partial metric space is a $\operatorname{pair}(X, p)$ such that $X$ is a nonempty set and $p$ is a partial metric on $X$.

Each partial metric $p$ on $X$ generates a $T_{0}$ topology $\tau_{p}$ on $X$ whose base is the family of open $p$-balls $\left\{B_{p}(x, \varepsilon), x \in\right.$ $X, \varepsilon>0\}$, where $B_{p}(x, \varepsilon)=\{y \in X: p(x, y)<p(x, x)+\varepsilon\}$ for all $x \in X$ and $\varepsilon>0$.

If $p$ is a partial metric on $X$, then the function $p^{s}: X \times$ $X \rightarrow R^{+}$given by

$$
p^{s}(x, y)=2 p(x, y)-p(x, x)-p(y, y)
$$

is a metric on $X$.

Definition 2. A mapping $f: X \rightarrow X$ is said to be continuous at $x_{0} \in X$, if for every $\varepsilon>0$, there exists $\delta>0$ such that $f\left(B_{p}\left(x_{0}, \delta\right)\right) \subset B_{p}\left(f\left(x_{0}\right), \varepsilon\right)$.

Definition 3 (see $[16])$. Let $(X, p)$ be a partial metric space and $\left\{x_{n}\right\}$ a sequence in $X$. Then,

(i) $\left\{x_{n}\right\}$ converges to a point $x \in X$ if $p(x, x)=$ $\lim _{n \rightarrow+\infty} p\left(x, x_{n}\right)$

(ii) $\left\{x_{n}\right\}$ is called a Cauchy sequence if $\lim _{n, m \rightarrow+\infty} p\left(x_{n}\right.$, $\left.x_{m}\right)$ exists and is finite.

Moreover, if $\lim _{m, n \rightarrow+\infty} p\left(x_{n}, x_{m}\right)=0$, then $\left\{x_{n}\right\}$ is said to be a 0 -Cauchy sequence in $(X, p)$.

Definition 4. A partial metric space $(X, p)$ is said to be complete if every Cauchy sequence $\left\{x_{n}\right\}$ in $X$ converges, with respect to $\tau_{p}$, to a point $x \in X$, such that $p(x, x)=$ $\lim _{n, m \rightarrow+\infty} p\left(x_{n}, x_{m}\right)$.
A partial metric space $(X, p)$ is called 0-complete [26] if every 0 -Cauchy sequence $\left\{x_{n}\right\}$ in $X$ converges to some $x \in X$ (with respect to $\tau_{p}$ ) such that $p(x, x)=0$. Therefore, $(X, p)$ is 0 -complete if and only if every 0 -Cauchy sequence converges with respect to $\tau_{p^{s}}$. It is clear that every 0 -Cauchy sequence in $(X, p)$ is a Cauchy sequence in $(X, p)$. Therefore, if $(X, p)$ is complete, then it is 0 -complete but not conversely (e.g., [26]).

In general, $p(x, y)$ is not a continuous function in two variables, in the sense that $x_{n} \rightarrow x$ and $y_{n} \rightarrow y$ (in $\tau_{p}$ ) imply that $p\left(x_{n}, y_{n}\right) \rightarrow p(x, y)$, as $n \rightarrow+\infty$. However, the following holds:

Lemma 5. Let $(X, p)$ be a partial metric space and $\left\{x_{n}\right\} \subset X$. If $x_{n} \rightarrow z$ as $n \rightarrow \infty$ and $p(z, z)=0$, then

$$
\lim _{n \rightarrow \infty} p\left(x_{n}, x\right)=p(z, x) \text {. }
$$

Lemma 6 (see $[16])$. Let $(X, p)$ be a partial metric space. Then,

(a) $\left\{x_{n}\right\}$ is a Cauchy sequence in $(X, p)$ if and only if it is a Cauchy sequence in the metric space $\left(X, p^{s}\right)$;

(b) $(X, p)$ is complete if and only if the metric space $\left(X, p^{s}\right)$ is complete. Furthermore, $\lim _{n \rightarrow+\infty} p^{s}\left(x_{n}, x\right)=0$ if and only if

$$
p(x, x)=\lim _{n \rightarrow+\infty} p\left(x_{n}, x\right)=\lim _{n, m \rightarrow+\infty} p\left(x_{n}, x_{m}\right) .
$$

Before proving our main result (i.e., Theorem 8), we state the following lemma available in $[5,13]$ for standard metric spaces. As the proof of the lemma is identical for the partial metrices, therefore, we merely state the lemma (for partial metric spaces).

Lemma 7. Let $(X, p)$ be a partial metric space and let $T$ be mapping on $X$. Let $x \in X$ satisfies $p\left(T x, T^{2} x\right) \leq r p(x, T x)$ for some $r \in[0,1)$. Then for $y \in X$, either

$$
\frac{1}{1+r} p(x, T x) \leq p(x, y) \text { or } \frac{1}{1+r} p\left(T x, T^{2} x\right) \leq p(T x, y)
$$

holds.

Now, we prove our main result as follows.

Theorem 8. Define a nonincreasing function $\psi$ from $[0,1)$ to $(1 / 2,1]$ by

$$
\psi(r)= \begin{cases}1, & 0 \leq r<\frac{1}{\sqrt{2}}, \\ \frac{1}{1+r}, & \frac{1}{\sqrt{2}} \leq r<1 .\end{cases}
$$

Let $(X, p)$ be a 0 -complete partial metric space and $T$ a selfmapping defined on $X$. Let $\gamma \in[0,1 / 2)$ and put $\lambda:=\gamma /(1-\gamma) \in$ $[0,1)$. If

$$
\begin{aligned}
& \psi(r) p(x, T x) \leq p(x, y) \\
& \quad \Longrightarrow p(T x, T y) \leq \gamma[p(x, T y)+p(y, T x)]
\end{aligned}
$$

for all $x, y \in X$, then $T$ has a unique fixed point. 
Proof. Since $\psi(r) \leq 1, \psi(r) p(x, T x) \leq p(x, T x)$ holds. In view of one of the hypotheses, we can write the following:

$$
\begin{aligned}
p\left(T x, T^{2} x\right) & \leq \gamma\left[p\left(x, T^{2} x\right)+p(T x, T x)\right] \\
& \leq \gamma\left[p(x, T x)+p\left(T x, T^{2} x\right)\right]
\end{aligned}
$$

so that

$$
p\left(T x, T^{2} x\right) \leq \frac{\gamma}{1-\gamma} p(x, T x)
$$

for $x \in X$. Now, fix $u_{0} \in X$ and define a sequence $\left\{u_{n}\right\}$ in $X$ by $u_{n}=T^{n} u_{0}=T u_{n-1}$. On using (8), we can have the following:

$$
\begin{aligned}
p\left(u_{n}, u_{n+1}\right)= & p\left(T^{n} u_{0}, T^{n+1} u_{0}\right) \\
\leq & \lambda p\left(T^{n-1} u_{0}, T^{n} u_{0}\right) \\
& \vdots \\
\leq & \lambda^{n} p\left(u_{0}, T u_{0}\right)
\end{aligned}
$$

so that

$$
\begin{aligned}
p\left(u_{n}, u_{m}\right) & \leq \frac{\lambda^{n}}{1-\lambda} p\left(u_{0}, T u_{0}\right) \longrightarrow 0 \\
\text { as } n & \longrightarrow \infty(\text { since } 0 \leq \lambda<1)
\end{aligned}
$$

for all $n, m \in \mathbb{N}$ and so $\left\{u_{n}\right\}$ is a 0 -Cauchy sequence. Since $(X, p)$ is 0 -complete, $\left\{u_{n}\right\}$ converges to some point $u \in X$; that is,

$$
\lim _{n \rightarrow \infty} p\left(u_{n}, u\right)=p(u, u) .
$$

Now, we show that

$$
p(u, T x) \leq \lambda p(x, T x) \quad \forall x \in X \backslash\{u\} .
$$

Since $u_{n} \rightarrow u$, there exists $n_{0} \in \mathbb{N}$ such that $p\left(u_{n}, u\right) \leq$ $p(u, x) / 3$ for all $n \geq n_{0}$. Owing to $p(x, u) \leq p\left(x, u_{n}\right)+p\left(u_{n}, u\right)$ and $p\left(u_{n}, u_{n+1}\right) \leq p\left(u_{n}, u\right)+p\left(u_{n+1}, u\right)$, for all $n \geq n_{0}$, we obtain the following:

$$
\begin{aligned}
\psi(r) p\left(u_{n}, T u_{n}\right) & \leq p\left(u_{n}, T u_{n}\right)=p\left(u_{n}, u_{n+1}\right) \\
& \leq p\left(u_{n}, u\right)+p\left(u_{n+1}, u\right) \\
& \leq \frac{2}{3} p(u, x)=p(u, x)-\frac{p(u, x)}{3} \\
& \leq p(u, x)-p\left(u_{n}, u\right) \\
& \leq p\left(u_{n}, x\right),
\end{aligned}
$$

and henceforth

$$
p\left(T u_{n}, T x\right) \leq \gamma\left[p\left(u_{n}, T x\right)+p\left(x, T u_{n}\right)\right] \quad \forall n \geq n_{0} .
$$

Now,

$$
\begin{aligned}
p(u, T x) & \leq \lim _{n \rightarrow \infty} p\left(u_{n+1}, T x\right)=\lim _{n \rightarrow \infty} p\left(T u_{n}, T x\right) \\
& \leq \lim _{n \rightarrow \infty} \gamma\left[p\left(u_{n}, T x\right)+p\left(x, T u_{n}\right)\right] \\
& =\lambda p(x, T x) \quad \text { (owing to Lemma } 5)
\end{aligned}
$$

for $x \in X$ with $x \neq u$. Let us prove that $u$ is a fixed point of $T$. In case $0 \leq r<1 / \sqrt{2}$, let on contrary that $T u \neq u$. Then owing to (12),

$$
\begin{aligned}
p\left(u, T^{2} u\right) & \leq \lambda p\left(T u, T^{2} u\right) \\
& \leq \gamma \lambda p(u, T u)
\end{aligned}
$$

so that

$$
\begin{aligned}
p(u, T u) & \leq p\left(u, T^{2} u\right)+p\left(T^{2} u, T u\right) \\
& \leq \gamma \lambda p(u, T u)+\gamma p(u, T u) \\
& \leq \frac{\gamma+2 \gamma^{2}}{1+\gamma} p(u, T u)<\frac{1+\gamma}{\gamma+1} p(u, T u) \\
& <p(u, T u),
\end{aligned}
$$

which is a contradiction. Therefore, we have $T u=u$. In case $1 / \sqrt{2} \leq r<1$, using Lemma 7 , we have the following:

$$
\begin{gathered}
\psi(r) p\left(u_{2 n}, u_{2 n+1}\right) \leq p\left(u_{2 n}, u\right) \\
\text { or } \quad \psi(r) p\left(u_{2 n+1}, u_{2 n+2}\right) \leq p\left(u_{2 n+1}, u\right)
\end{gathered}
$$

for $n \in \mathbb{N}$. Thus, there exists a subsequence $\left\{n_{j}\right\}$ of $\{n\}$ such that

$$
\psi(r) p\left(u_{n_{j}}, u_{n_{j}+1}\right) \leq p\left(u_{n_{j}}, u\right)
$$

for $j \in \mathbb{N}$. On using one of our assumption, we have the following:

$$
\begin{aligned}
p(u, T u) & =\lim _{j \rightarrow \infty} p\left(u_{n_{j}+1}, T u\right) \\
& \leq \lim _{j \rightarrow \infty} \gamma\left[p\left(u_{n_{j}}, T u\right)+p\left(u, T u_{n_{j}}\right)\right] \\
& =\gamma p(u, T u) .
\end{aligned}
$$

As $\gamma<1 / 2$, we have $T u=u$. The uniqueness of fixed point is obvious in view of (12). This concludes the proof.

Notice that, in the course of proving Theorem 9 below, we have shown the following: (i) $(X, p)$ is a complete partial metric space, (ii) $T$ satisfies condition (21) but has no fixed point.

The following theorem shows that $\psi(r)$ is the best constant for every value of $r$.

Theorem 9. Define a function $\psi$ as in Theorem 8. For every $\gamma \in[0,1 / 2)$, setting $\lambda:=\gamma /(1-\gamma)$, there exists a 0 -complete partial metric space $(X, p)$ and a mapping $T$ on $X$ such that $T$ has no fixed point but

$$
\begin{aligned}
& \psi(r) p(x, T x)<p(x, y) \\
& \quad \Longrightarrow p(T x, T y) \leq \gamma[p(x, T y)+p(y, T x)]
\end{aligned}
$$

for all $x, y \in X$. 
Proof. Let $X=[0,1] \cup[2,3]$ and define a complete partial metric $p$ on $X$ by the following:

$$
p(x, y)= \begin{cases}|x-y|, & \{x, y\} \subset[0,1], \\ \max \{x, y\}, & \{x, y\} \cap[2,3] \neq \emptyset .\end{cases}
$$

Then $(X, p)$ is obviously 0 -complete. Define a mapping $T$ on $X$ by the following:

$$
T x= \begin{cases}1, & 0 \leq x<1, \\ \frac{3}{4}, & x=1, \\ \frac{1}{4}, & 2 \leq x \leq 3 .\end{cases}
$$

Notice that $T$ has no fixed point.

Firstly if $0 \leq r<1 / \sqrt{2}$, then the following cases arise.

(i) If $x=y=0, x=y=1, x=y=2$, and $x=y=$ 3 , then $T x=T y$. Hence, the right-hand side of (21) trivially holds so that implication is also verified.

(ii) If $x=1, y=0$, then $T x=3 / 4, T y=1$ and hence the left-hand side of (21) holds good as $\psi(r) p(x, T x)=$ $1 / 4<1=p(x, y)$. Also, the right-hand side of (21) is satisfied in view of the following:

$$
\begin{aligned}
p(T x, T y) & =p(T 1, T 0)=\frac{1}{4} \\
& \leq \gamma[p(x, T y)+p(y, T x)]=\frac{3}{4} \gamma
\end{aligned}
$$

as there exists such a $\gamma \in[0,1 / \sqrt{2})$.

(iii) If $x=0, y=1$, then $T x=1, T y=3 / 4$ and hence the right-hand side of (21) trivially holds so that implication is also realized.

(iv) If $\{x, y\} \subset(0,1)$, then $T x=T y=1$ so that the left-hand side of (21) holds good as $\psi(r) p(x, T x)=$ $|x-1|<|x-y|=p(x, y)($ as $y<1)$. The righthand side of (21) also remains true as $p(T x, T y)=0 \leq$ $\gamma[p(x, T y)+p(y, T x)]$.

(v) If $\{x, y\} \cap(2,3) \neq \emptyset$, then $T x=T y=1 / 4$, wherein we examine two cases. If $x \geq y$, then the righthand side of (21) trivially holds and hence so does the implication. If $x<y$, then the left-hand side of (21) remains true as $\psi(r) p(x, T x)=x<p(x, y)$. The right-hand side of (21) also holds good as

$$
p(T x, T y)=0 \leq \gamma[p(x, T y)+p(y, T x)] .
$$

Secondly, if $1 / \sqrt{2} \leq r<1$, then we distinguish the following cases.

(i) If $x=y=0, x=y=1, x=y=2$, and $x=y=3$, then $T x=T y$. Hence, the right-hand side of (21) as well as implication remains true. (ii) If $\{x, y\} \subset(0,1)$, then $T x=T y=1, p(x, T x)=$ $|x-1|$. The left-hand side of (21) holds good as $\psi(r) p(x, T x)=(1 /(r+1))|x-1|<|x-y|$. The righthand side of (21) remains true as

$$
p(T x, T y) \leq \gamma[p(x, T y)+p(y, T x)] .
$$

(iii) If $x=0, y=1$, then $T x=1, T y=3 / 4$. The left-hand side of (21) remains true as $\psi(r) p(x, T x)=1 /(r+1)<$ $1=p(x, y)$, The right-hand side of (21) is satisfied as follows:

$$
\begin{aligned}
p(T x, T y) & =\frac{1}{4} \leq \gamma \frac{3}{4} \\
& \leq \gamma[p(x, T y)+p(y, T x)]
\end{aligned}
$$

if we choose $\gamma \geq 1 / 3$.

(iv) If $x=1, y=0$, then $T x=3 / 4, T y=1$. The lefthand side of (21) holds good as $\psi(r) p(x, T x)=(1 /(r+$ 1)) $(1 / 4)<1=p(x, y)$. The right-hand side of (21) is also satisfied as follows:

$$
\begin{aligned}
p(T x, T y) & =\frac{1}{4} \leq \gamma \frac{3}{4} \\
& \leq \gamma[p(x, T y)+p(y, T x)]
\end{aligned}
$$

if we can choose $\gamma \geq 1 / 3$.

(v) If $\{x, y\} \cap(2,3) \neq \emptyset$, then $T x=T y=1 / 4$. If $x<$ $y$, then the left-hand side of (21) remains true as $\psi(r) p(x, T x)=(1 /(1+r)) x<y=p(x, y)$. The righthand side of (21) is also verified as follows:

$$
p(T x, T y)=0 \leq \gamma[p(x, T x)+p(y, T y)] .
$$

If $x \geq y$, then left-hand side of (21) holds good as $\psi(r) p(x, T x)=(1 /(1+r)) x<x=p(x, y)$. The righthand side of (21) remains true as

$$
p(T x, T y)=0 \leq \gamma[p(x, T x)+p(y, T y)] .
$$

Thus, in all possible cases the condition (21) is satisfied.

\section{Acknowledgment}

Both the authors are grateful to both the learned referees for their fruitful comments towards the improvement of this paper.

\section{References}

[1] S. Banach, "Sur les opérations dans les ensembles abstraits et leur application aux équations intégrales," Fundamenta Mathematicae, vol. 3, pp. 133-181, 1922.

[2] W. A. Kirk, "Caristi's fixed point theorem and metric convexity," Colloquium Mathematicum, vol. 36, no. 1, pp. 81-86, 1976.

[3] P. V. Subrahmanyam, "Completeness and fixed-points," Monatshefte für Mathematik, vol. 80, no. 4, pp. 325-330, 1975. 
[4] E. H. Connell, "Properties of fixed point spaces," Proceedings of the American Mathematical Society, vol. 10, pp. 974-979, 1959.

[5] T. Suzuki, "A generalized Banach contraction principle that characterizes metric completeness," Proceedings of the American Mathematical Society, vol. 136, no. 5, pp. 1861-1869, 2008.

[6] O. Popescu, "Fixed point theorem in metric spaces," Bulletin of the Transilvania University of Braşov III, vol. 1, no. 50, pp. 479482, 2008.

[7] I. Altun and A. Erduran, "A Suzuki type fixed-point theorem," International Journal of Mathematics and Mathematical Sciences, vol. 2011, Article ID 736063, 9 pages, 2011.

[8] J. T. Markin, "A fixed point theorem for set valued mappings," Bulletin of the American Mathematical Society, vol. 74, pp. 639640, 1968.

[9] S. B. Nadler, "Multi-valued contraction mappings," Pacific Journal of Mathematics, vol. 30, pp. 475-488, 1969.

[10] M. Kikkawa and T. Suzuki, "Three fixed point theorems for generalized contractions with constants in complete metric spaces," Nonlinear Analysis. Theory, Methods \& Applications Series A: Theory and Methods, vol. 69, no. 9, pp. 2942-2949, 2008.

[11] Y. Enjouji, M. Nakanishi, and T. Suzuki, "A generalization of Kannan's fixed point theorem," Fixed Point Theory and Applications, vol. 2009, Article ID 192872, 10 pages, 2009.

[12] O. Popescu, "Two fixed point theorems for generalized contractions with constants in complete metric space," Central European Journal of Mathematics, vol. 7, no. 3, pp. 529-538, 2009.

[13] M. Kikkawa and T. Suzuki, "Some similarity between contractions and Kannan mappings," Fixed Point Theory and Applications, vol. 2008, Article ID 649749, 8 pages, 2008.

[14] M. Nakanishi and T. Suzuki, "An observation on Kannan mappings," Central European Journal of Mathematics, vol. 8, no. 1, pp. 170-178, 2010.

[15] O. Popescu, "A new type of contractive multivalued operators," Bulletin des Sciences Mathématiques, vol. 137, no. 1, pp. 30-44, 2013.

[16] S. G. Matthews, "Partial metric topology", in Procedings of the 8th Summer Conference on General Topology and Applications, vol. 728 of Annals of the New York Academy of Sciences, pp. 183197, 1994.

[17] R. Heckmann, "Approximation of metric spaces by partial metric spaces," Applied Categorical Structures, vol. 7, no. 1-2, pp. 71-83, 1999.

[18] R. D. Kopperman, S. G. Matthews, and H. Pajoohesh, "What do partial metrics represent?" in Proceedings of the 19th Summer Conference on Topology and Its Applications, University of Cape Town, 2004.

[19] R. Kopperman, S. Matthews, and H. Pajoohesh, "Partial metrizability in value quantales," Applied General Topology, vol. 5, no. 1, pp. 115-127, 2004.

[20] H. P. A. Künzi, H. Pajoohesh, and M. P. Schellekens, "Partial quasi-metrics," Theoretical Computer Science, vol. 365, no. 3, pp. 237-246, 2006.

[21] S. J. ONeill, "Two topologies are better than one," Tech. Rep., University of Warwick, Coventry, UK, 1995, http://www .dcs.warwick.ac.uk/reports/283.html.

[22] M. P. Schellekens, "A characterization of partial metrizability: domains are quantifiable," Theoretical Computer Science, vol. 3 , pp. 91-112, 2002.
[23] O. Valero, "On Banach fixed point theorems for partial metric spaces," Applied General Topology, vol. 6, no. 2, pp. 229-240, 2005.

[24] S. Oltra and O. Valero, "Banach's fixed point theorem for partial metric spaces," Rendiconti dell'Istituto di Matematica dell'Università di Trieste, vol. 36, no. 1-2, pp. 17-26, 2004.

[25] I. Altun, F. Sola, and H. Simsek, "Generalized contractions on partial metric spaces," Topology and its Applications, vol. 157, no. 18, pp. 2778-2785, 2010.

[26] S. Romaguera, "A Kirk type characterization of completeness for partial metric spaces," Fixed Point Theory and Applications, vol. 2010, Article ID 493298, 6 pages, 2010.

[27] D. Paesano and P. Vetro, "Suzuki's type characterizations of completeness for partial metric spaces and fixed points for partially ordered metric spaces," Topology and its Applications, vol. 159, no. 3, pp. 911-920, 2012. 


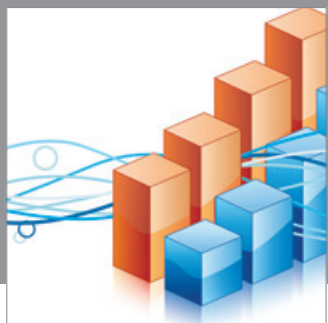

Advances in

Operations Research

mansans

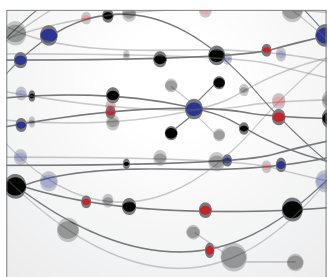

The Scientific World Journal
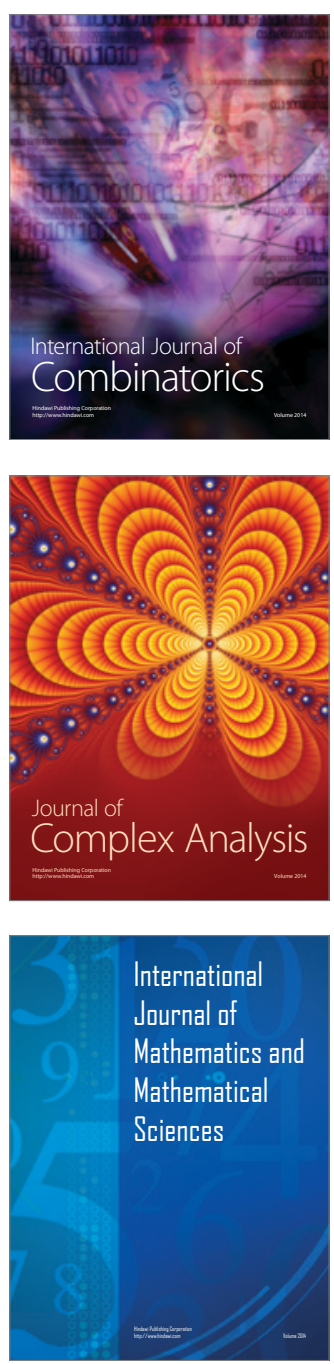
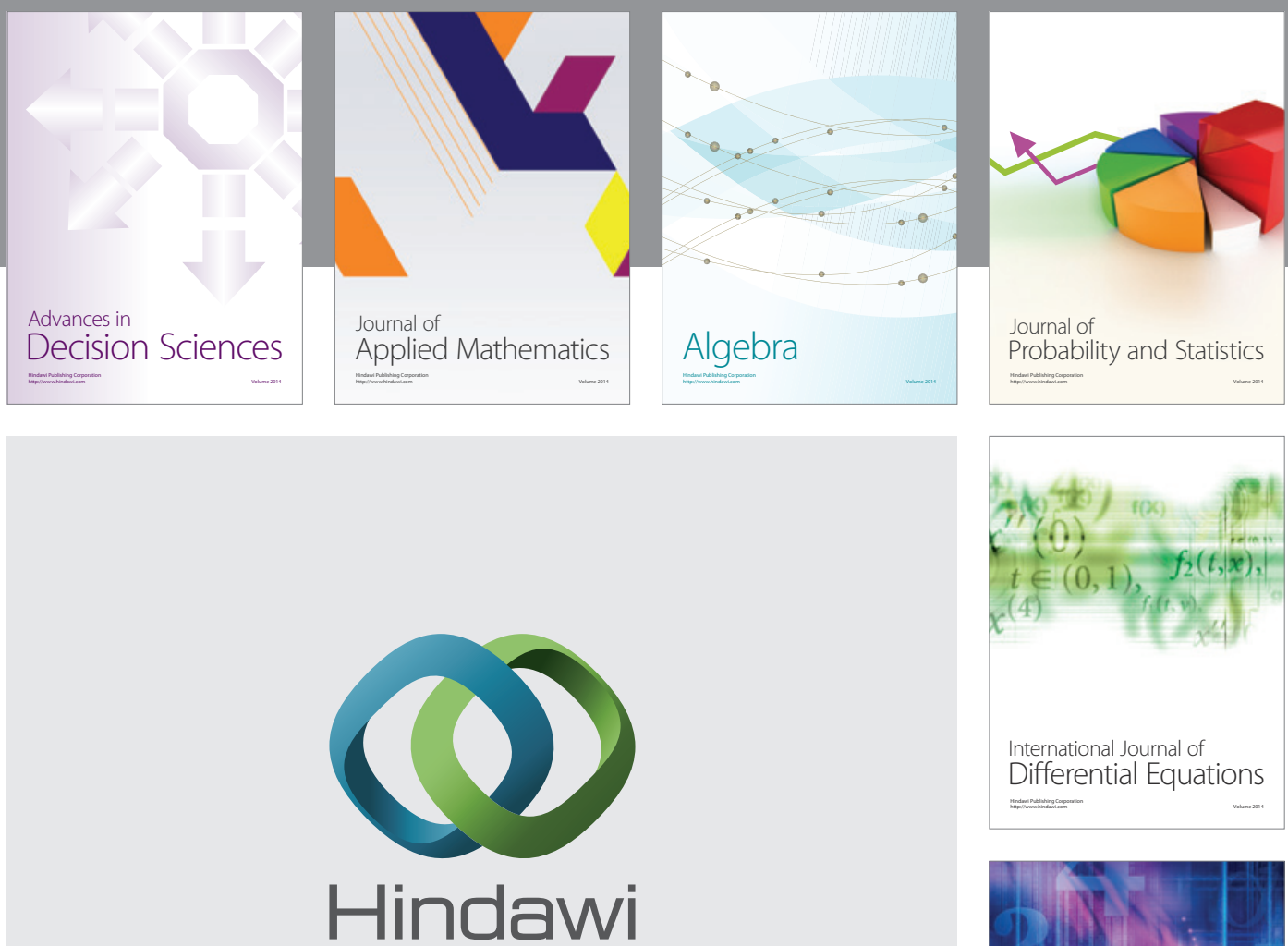

Submit your manuscripts at http://www.hindawi.com
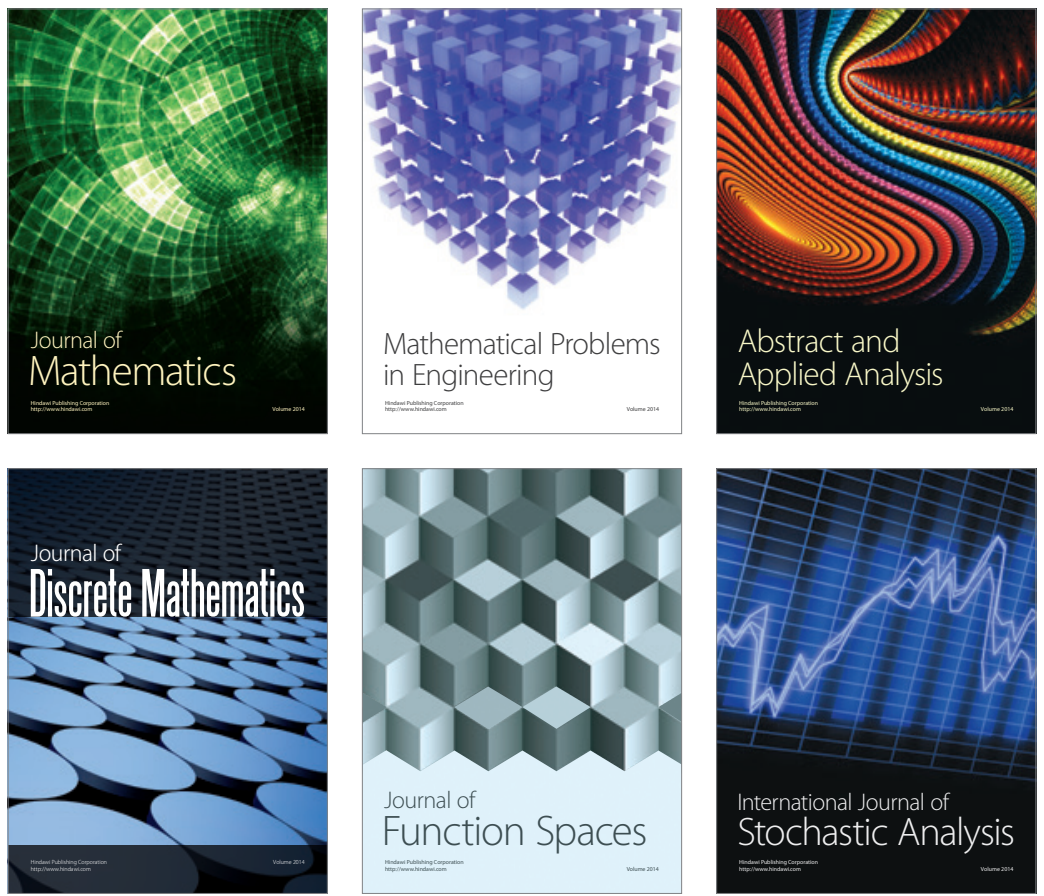

Journal of

Function Spaces

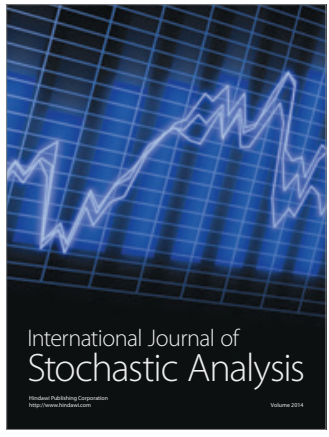

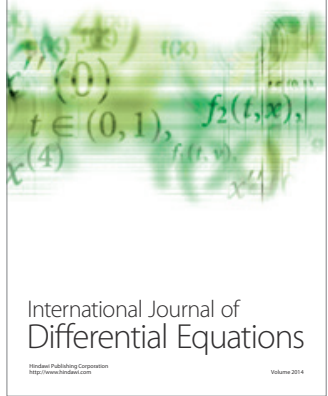
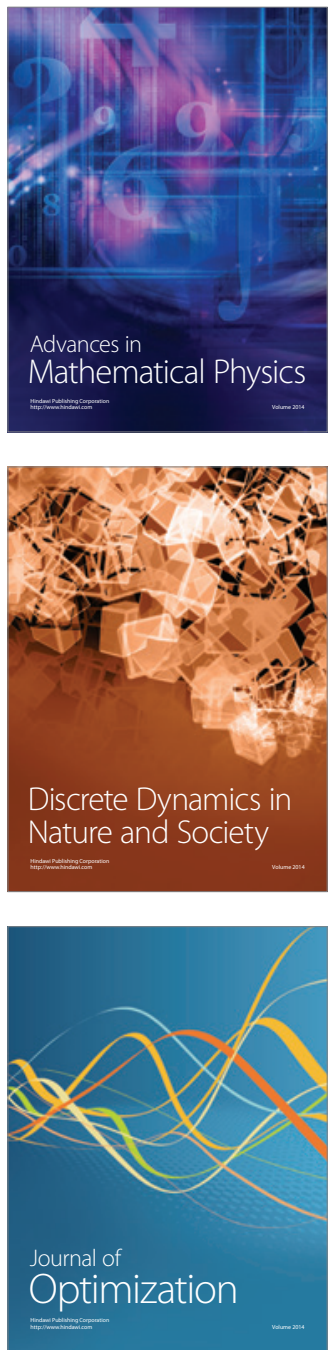